\title{
DESIGN AND QUALITY ASSURED MANUFACTURING OF FREE FORM METAL PROSTHESES BY SELECTIVE LASER MELTING TECHNOLOGY
}

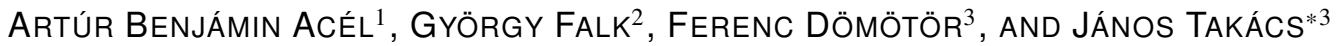 \\ ${ }^{1}$ eCon Engineering Kft., Kondorosi út 3, Budapest, 1116, HUNGARY \\ ${ }^{2}$ VARINEX Informatics Inc., Fehér út 10, Budapest, 1106, HUNGARY \\ ${ }^{3}$ Department of Automotive Technologies, Budapest University of Technology and Economics, Stoczek u. 4, \\ Budapest, 1111, HUNGARY

\begin{abstract}
This paper concerns the key steps in the design, manufacturing and certification of customized acetabular cups. The process is based on the compilation of computed tomography scans to create a surface that is generated from the point cloud of the surface model. As a result, the surface model is obtained. The final step is the manufacturing itself. The features of Selective Laser Melting, also referred to as Direct Metal Laser Sintering in the literature, the placement of workpieces in the construction space and peculiarities of the support design are described. Important technological preparations of the EOS M 100 3D camera for the manufacturing of implants will be described. Implants were made of the $316 \mathrm{~L}$ and Ti6Al4V metal powders. The finished test pieces were subjected to non-destructive as well as destructive mechanical and material structural testing to qualify implants by using the appropriate quality assurance system.
\end{abstract}

Keywords: 3D printing, computed tomography, metal prostheses

\section{Introduction}

Recently, the level of development of additive manufacturing has advanced. During the first level, the basics of the technology were elaborated on before the various fields of application were discovered during the following stage. Barriers have been removed and the application has become more and more effective. In the clinical and medical sciences, several companies have been trying to replace traditional implants with those produced by additive manufacturing. Although the imagination of designers in the field of engineering technology has no limits, limitations are imposed by medical doctors. Nowadays, individual implants can be applied in many individual cases without any complications [4]. Our paper describes the full sequence of preparing a real, customized acetabular cup.

\section{Tailor-made human implant}

\subsection{Accessing the necessary input data}

Unfortunately, the scapula of a woman was attacked by bone cancer. Since important muscles join to its surface, it is vital that this bone be replaced. Using computed tomography (CT) and magnetic resonance imaging (MRI)

*Correspondence: janos.takacs@gjt.bme.hu

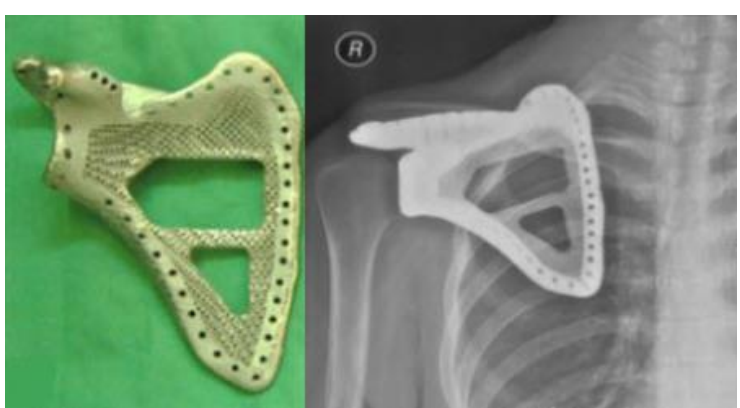

Figure 1: Tailor-made implant to replace the scapula [1]

images, engineers and medical doctors were able to reconstruct the bone to be replaced. The implant before and after implantation can be seen in Fig. 1. [1]

The left-hand side of Fig. 1 shows that the implant can be lightened or the solid (dense) material replaced by a barred structure. To properly attach the muscles at the edge of the implants, suitable connections can be created. Even in this case, the implant was fixed properly; it sat properly and the patient did not exhibit any symptoms after the operation. This case study can form the basis for proving that implants are useful and important. [1]

\subsection{DICOM to STL conversion}

Tailor-made requirements were formulated during a meeting between a doctor and engineer. As a first step, 


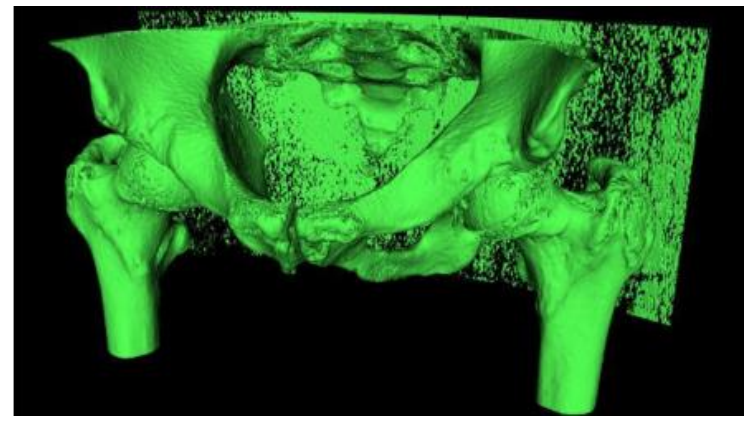

Figure 2: The object model compiled from CT scans

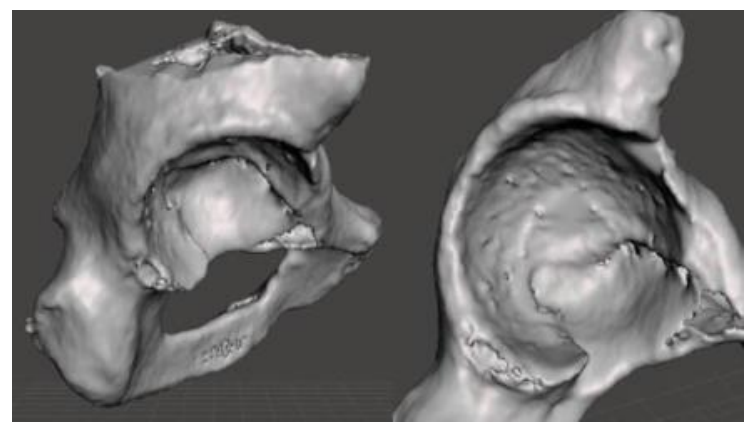

Figure 3: The "cleaned" surface model and the surface the socket must be adjusted to

the doctor has to submit proper records stored on CDs to the engineer. These records are usually stored on CDs or DVDs that can be read by any CD/DVD drive. The InVesalius software carries out the conversion, which is very user-friendly. The surface model for the socket to be manufactured, prepared by InVesalius software, can be seen in Fig. 2.

Given that the model generated by the InVesalius software can be imported and the surfaces that are unnecessary for designing implants removed from the surface model, the surface model is simpler and the storage of big files is not required. The Autodesk Meshmixer software can carry out this type of cleaning. In Fig. 3, the part of the surface where the implant has to be inserted can be seen. The background produced by the CT scanner on this surface model was removed from the femur and one half of the hip bone. During the next step, the file of the model had to be saved as an "STL" file to create the surface from triangles before proceeding with the design.

\subsection{Creation of the socket model}

If the corners of the triangles can be determined and substituted by points, a point cloud is produced. This point cloud has to be cleaned further, moreover, for the remaining points, a surface can be adjusted. By highlighting the adjusted surface, the shape and volume model of the socket is obtained. The Rhino 6 program can do this task. The point cloud generated from the saved surface model can be seen in Fig. 4.

It is also possible to simply delete any unnecessary points. During this step, how to fix the implant and what

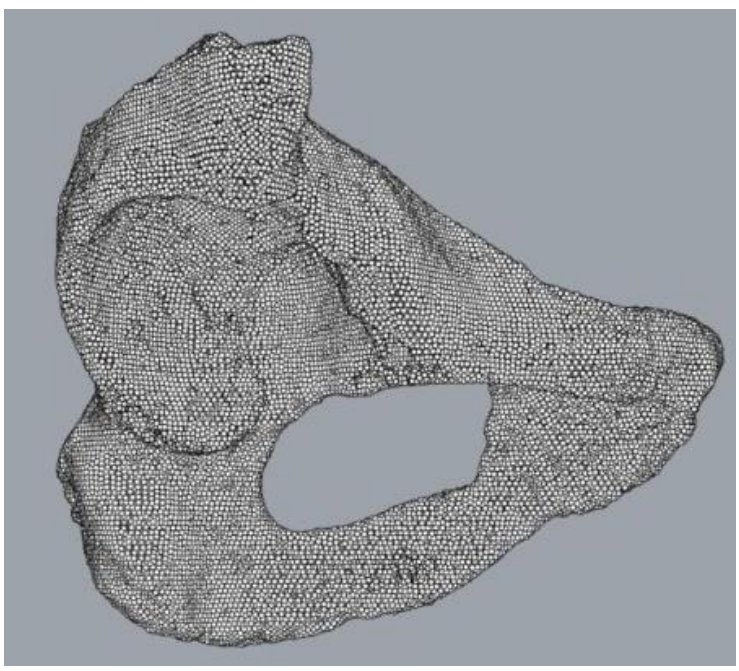

Figure 4: The point cloud generated from the "STL" file

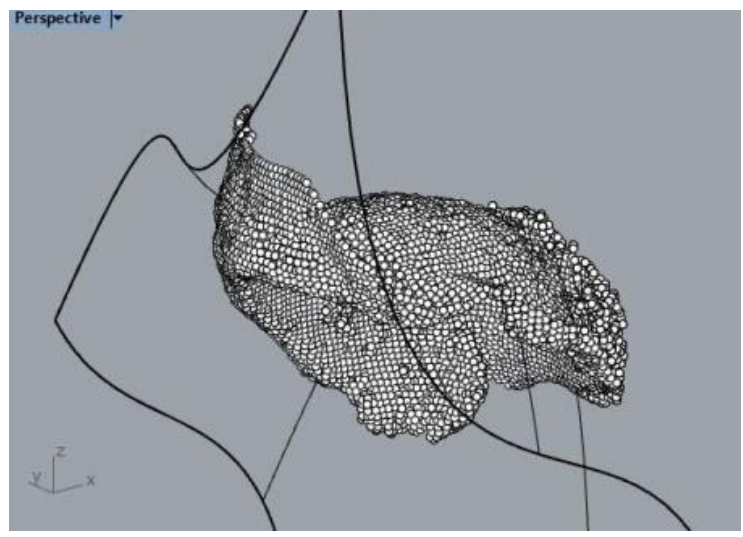

Figure 5: Generated surface with an insufficient number of control points

its role will be must be taken into account. It is important to mention that the fixed points cannot be determined from the CT images. The location of the screws in the bones is based on the experience of medical professionals.

The general purpose of the socket is to compensate for the missing bone that disintegrated on the surface of the femur. It is important that it can be fixed in several ways, e.g. the volume of the implant is cavernous so the bone can grow into the holes of the bones.

Bolts are used to fix traditional implants. This implant is designed to be fixed to the highly strong hip bone. At least three fastening points are required to safely fasten the socket while ensuring that a sufficient amount of space remains for the bolts.

By taking into account all of the aforementioned points, the point cloud can be approximated by a surface. The program must follow set conditions to determine how many control points the surface should be generated from. In this case, $20 \times 20$ was determined as suitable. If the number of control points is any less, the point cloud cannot be simulated properly. This case is shown in Fig. 5. 


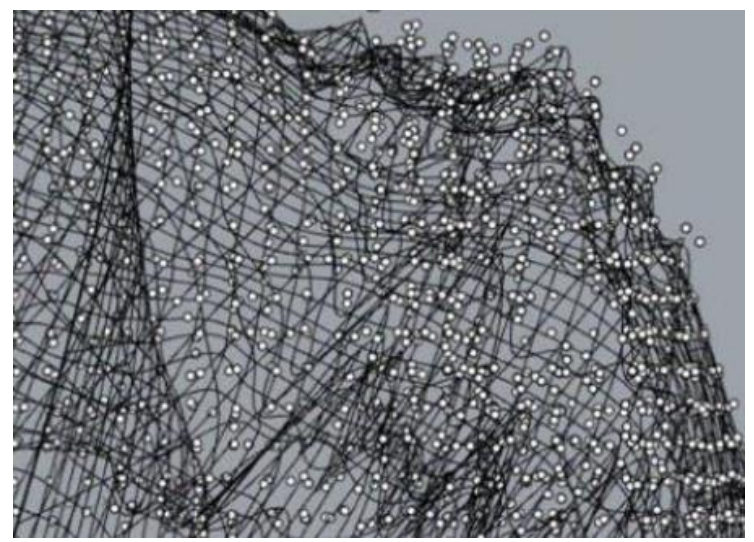

Figure 6: The superfluous points of the point cloud are shown in detail on the generated surface

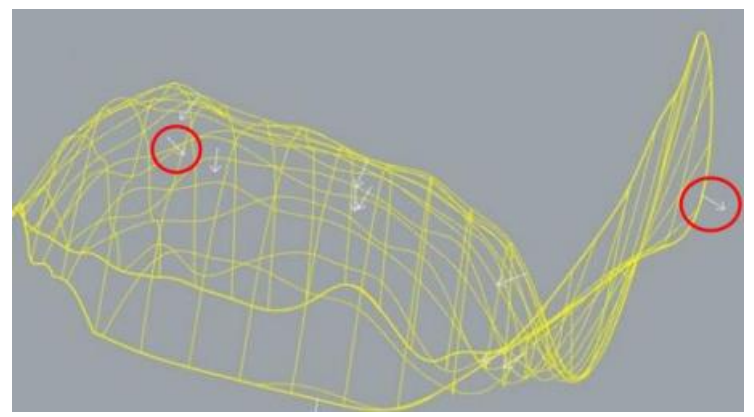

Figure 7: After determining the limitations of the socket, the directions of the normal vectors of the surface are designated by red circles.

As can be seen in Fig. 6, should too many control points be present, the program shows the undesirable defects of the surface that result. When the surface is ready, then the point cloud can be removed. The boundaries of the surface are not determined. The control points cannot be given in such a way that they reach the boundary of the point cloud.

The limitations of the implant have to be determined. In this piece of software, it is possible to reshape the surface that results. Predetermined sections can be projected onto the surface. Using these projected sections, the individual parts of the surface can be detached, as can be seen in Fig. 7.

After forming the required shape, it is possible to stretch the surface in the direction of its normal vector to create the required thickness of the wall. It should be noted that this surface will make contact with the bone, so must be stretched in the opposite direction. The stretched surfaces can be seen in Fig. 8. It is important that the surface model forms a closed surface to imitate the body. This closed surface model can be transferred into a body using the Rhino 6 program. This model of a body can be stored in an "STP" file format. Any traditional CAD program is capable of opening this format.

As the next step, the holes for fixing the bolts have to be created in the CAD system before the model of the body can be prepared for manufacturing and its verifica-

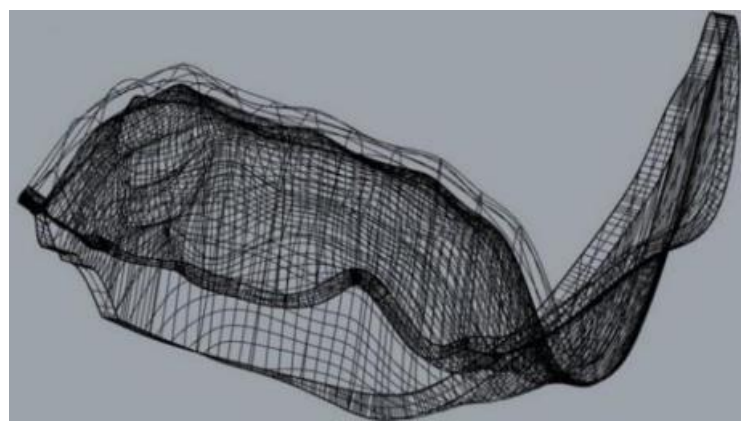

Figure 8: The final closed surface model after being stretched in the direction of the normal vector

tion.

\subsection{Powder bed fusion for the additive manu- facturing of metal parts using laser beams}

Selective Laser Melting, similarly to other additive manufacturing technologies like Direct Metal Laser Sintering, constructs the workpiece layers by layer. At the Department of Automotive Technologies at BME, a special method has been developed for the EOS M 100 3D camera with the following operating principles:

- The first step is to build a layer (on a heated base plate) that sinks proportionally to the thickness of the powder.

- The second step is to provide a dose of powder from the automated supply equipment.

- The third step is to spread the dose of powder over the work surface and push the superfluous amount into the container located on the other side of the work surface.

- The fourth step is to scan the laser beam over the work surface before melting and fastening the particles of powder. The melting will result in the thickness of the layer of powder decreasing.

- The fifth step is that the recoter returns to its original position (end position on the left-hand side) before being prepared for the portioning.

Four doses (denoted as 1-2-3-4) can be set on the working set and spread by the working blade to a thickness of 20 $\mu \mathrm{m}$.

\section{Positioning of the workpiece in the building space}

Should several workpieces be present, their positions are important. The working blade must spread the powder smoothly and any fluctuations of forces acting on the blade must be avoided since these might cause vibrations leading to variations in the thickness of the powder.

Another problem can occur if the workpieces are arranged in a row because the separated small workpieces 


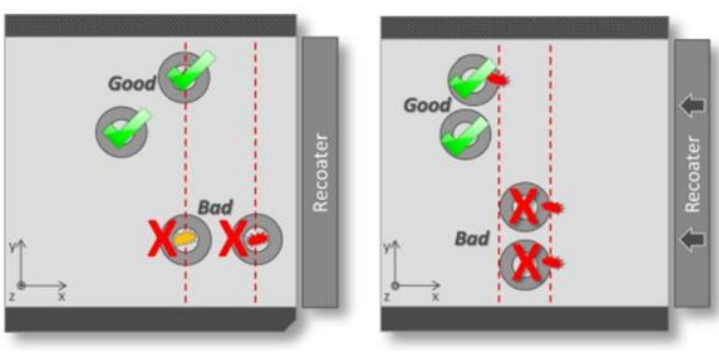

Figure 9: An optional advantageous arrangement of the workpieces [2]

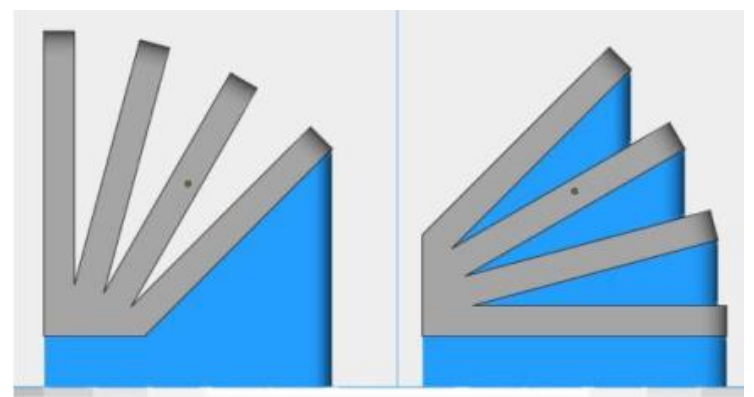

Figure 10: Generation of a support (denoted in blue) depending on the angle of incidence [3]

might jam the blade. An optional advantageous arrangement can be seen in Fig. 9.

According to our experience, small workpieces should not be placed on an edge because the temperature distribution here is not uniform which can cause problems.

\section{Design of a workpiece support on the heated base plate}

Proper binding of the initial layer of the workpiece is essential, otherwise the support will be subjected to thermal stress due to differences in temperature. If the support is not fastened properly to the base plate, then the workpiece might be torn and, consequently, production might have to be stopped. Another important factor is heat transfer and cooling because the workpiece can burn due to a prolonged period at high temperatures resulting in failure of the surface and structural features. The Materialise Magics program can design a support for the workpieces. The following recommendations should be followed.

It is undesirable to leave the surface unsupported if the angle of incidence is more than 45 degrees (Fig. 10).

Bulging, overhanging parts also have to be supported. They might become superfluous as well because the workpiece can be placed in different orientations in the workspace.

\section{Preparation for the manufacturing of a tailor-made socket}

As a first step, the workpiece has to be imported into the Materialise Magics program before being placed in the

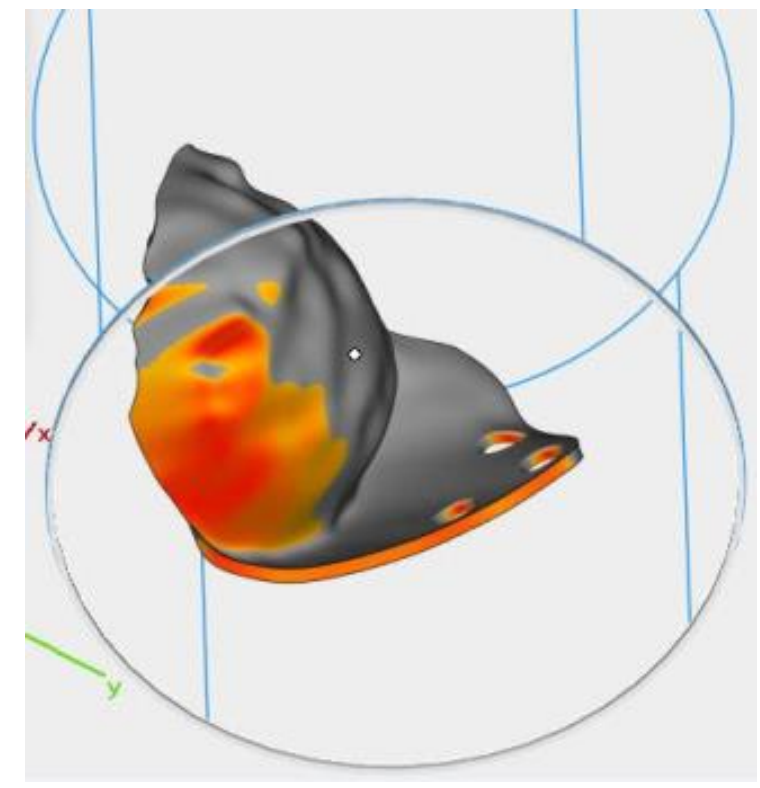

Figure 11: Bottom view of the surfaces with a maximum angle of declination of 45 degrees.

proper position. It is advisable to minimize the number of supports. Once the model has been placed in the building space, it can be adjusted into its final position. For this purpose, the module "Supported area preview" is an excellent tool providing coloured data about the steepness of the surfaces. The location of the socket can be seen in Fig. 11.

The next step is to partition common supports by following the command "fragmentation," resulting in a reduction in the total manufacturing time because no additional supports are required and consequently less powder is needed. Lightening of load-bearing walls means the holes should be diamond-shaped or rectangular. This enables powder which is not molten to be cleared from the load-bearing walls. After clearing, the superfluous material can easily be removed and the construction time reduced because the laser no longer needs to scan the vector section. "Fragmentation" and lightening of the diamond shape can be seen in Fig. 12.

The so-called "teeth" connect the workpiece to the supports. Since the program separates the workpiece and the supports, it is no longer necessary to scan the teeth supporting the workpiece. Ingrowing can be prevented by following the "Z Offset" command as shown in Fig. 13.

\subsection{Checking the manufacturing of the pre- pared socket model}

Due to thermal deformation during the manufacturing process, the blade may get jammed in the workpiece. Furthermore, it is possible that the workpiece will crack due to the tension. The production process will stop in both cases due to overloading of the blade. To avoid this, the manufacturing plan has to be checked. Naturally, the signals have to be verified and taken into account or manually neglected by the supervisor. 


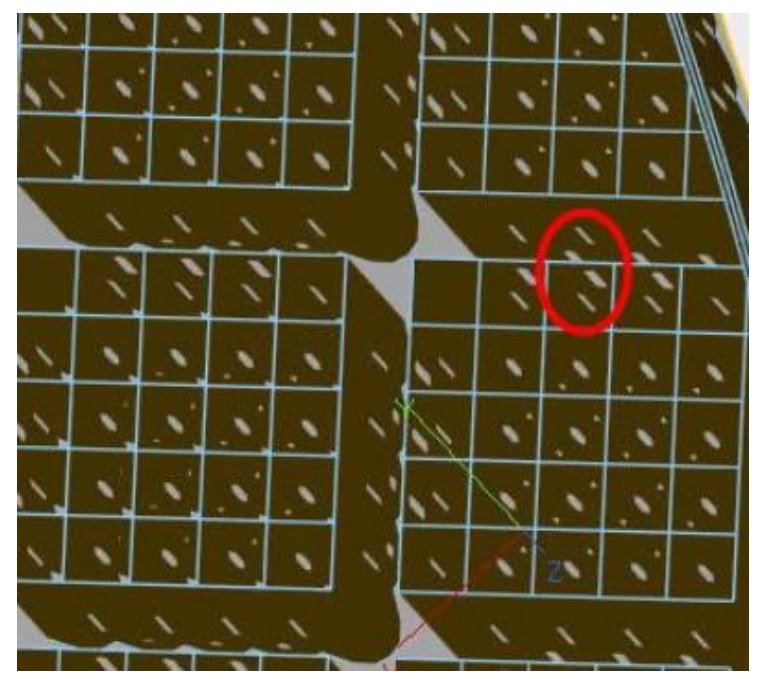

Figure 12: "Fragmentation" and lightening of the diamond shape highlighted by a red circle

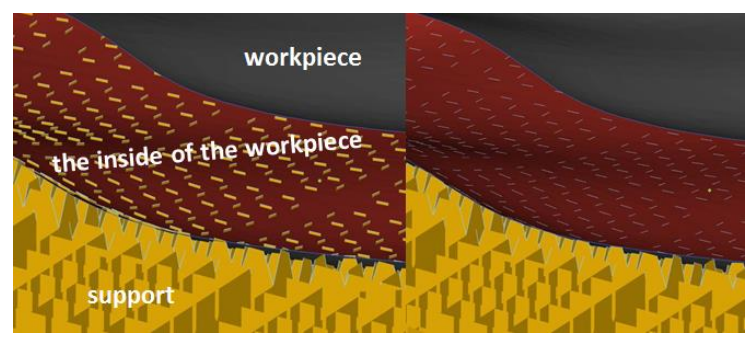

Figure 13: Prevention of the ingrowing of the teeth on the workpiece

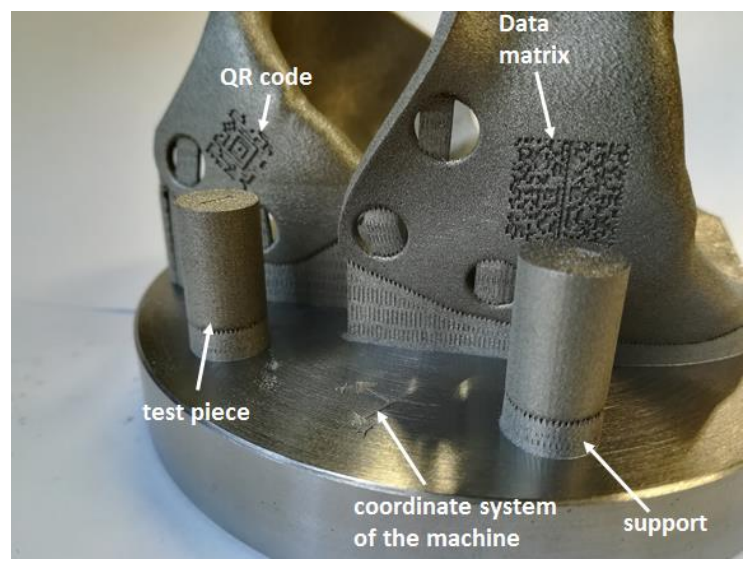

Figure 14: Sockets made of the alloy $316 \mathrm{~L}$

The building platform is fastened in place by a vacuum system. The zero positions in the vertical direction must be fixed and the whole building surface levelled to achieve the required degree of flatness. The powder container must be filled and pushed into place. The protective cover must be cleaned with alcoholic tissue paper before manufacturing. The next step is to cover the door of the workspace. The entire manufacturing process takes place in an atmosphere of inert argon gas.

Two sockets were designed. The workspace was filled with $99.99 \%$ argon gas, which is heavier than air so fills

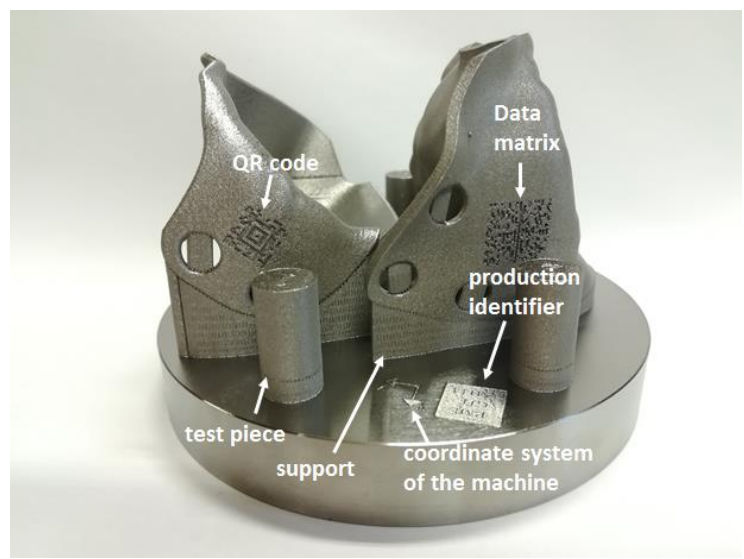

Figure 15: Sockets made of the alloy Ti6A14V

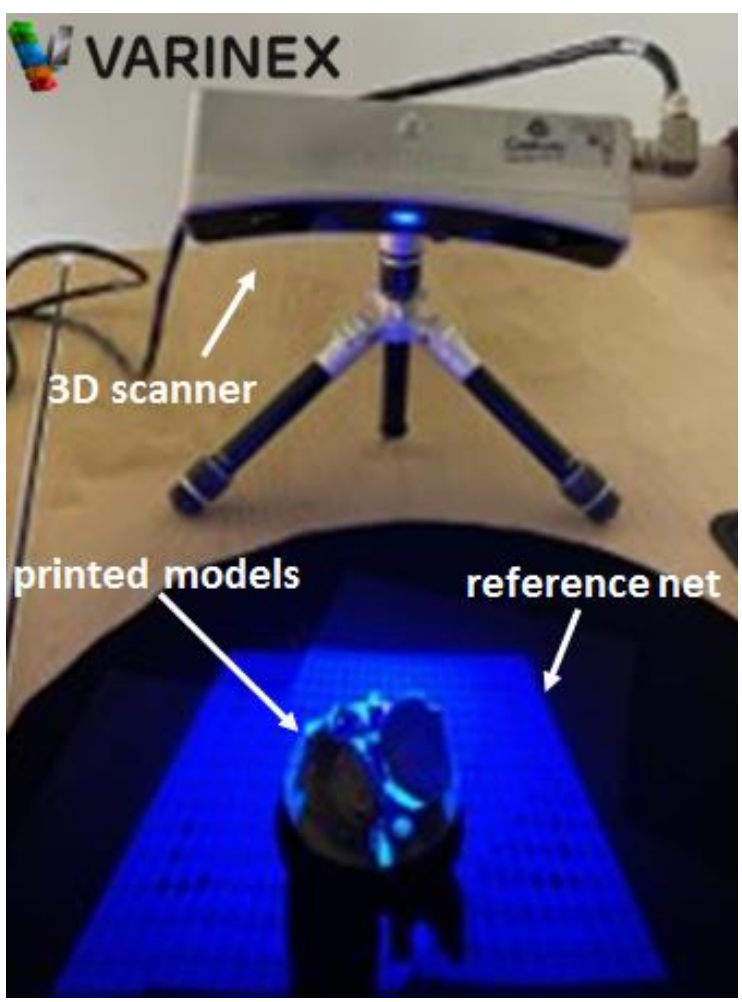

Figure 16: Measurement arrangement of the 3D Systems Capture scanner the space from the bottom up. If less than $0.1 \%$ of oxygen is present, then the manufacturing process can commence. During the first manufacturing process, the designed sockets were made of the alloy $316 \mathrm{~L}$. One of the sockets was designed by using the Simufact program to facilitate geometrical measurements. In the manufacturing workspace, more trial workpieces were placed. The sockets were manufactured successfully, as can be seen in Fig. 14. During the second manufacturing process, the sockets were made of the alloy Ti6A14V supplied by EOS and can be seen in Fig. 15. 


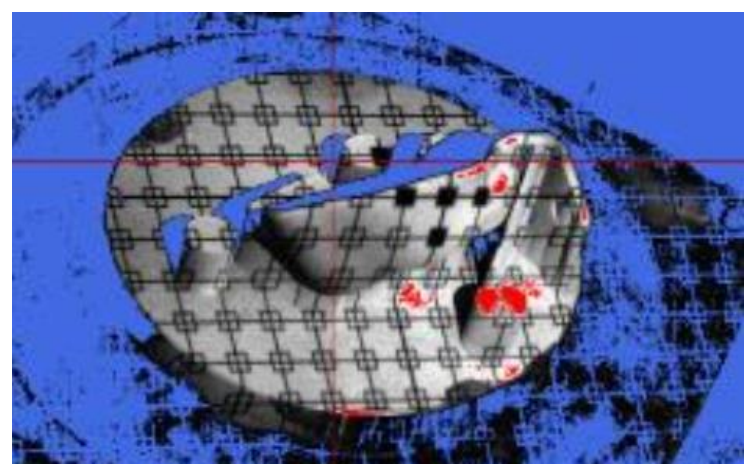

Figure 17: Surface with the reference net after being scanned by the $3 \mathrm{D}$ camera

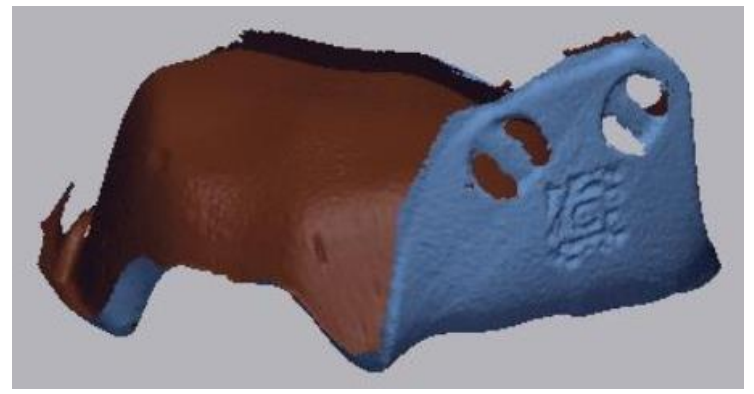

Figure 18: Surface model after smoothing

\section{Evaluation and quality control by the ge- ometrical measurements}

VARINEX Informatics Inc. carried out the geometrical measurements using the scanner presented in Fig. 16.

Before the measurements were taken, the workpieces were sprayed in a "processing powder" to avoid glittering. The workpieces to be measured are placed at the centre of the reference net. The exposition time has to be adjusted according to the light conditions.

During the scanning, two images of the workpiece are made before the program generates a powder cloud of the surface as can be seen in Fig. 17.

Surfaces scanned from several directions have to be compiled manually because the forms are unusual and the program is unable to smoothen these surfaces, which can be seen in Fig. 18. Unfortunately, due to the support, one part of the surface could not be scanned.

This surface model can be compared with the original CAD model using the Geomagic Design X program. The socket models were scanned in raw, heat-treated and cut statuses. These surfaces were compared with the original CAD geometry and the results can be seen in Fig. 19. The limits of measurements during the comparison were between $1 \mathrm{~mm}$ and $-1 \mathrm{~mm}$. Differences in size are denoted by colours. The maximum values and distributions are also presented in this image.

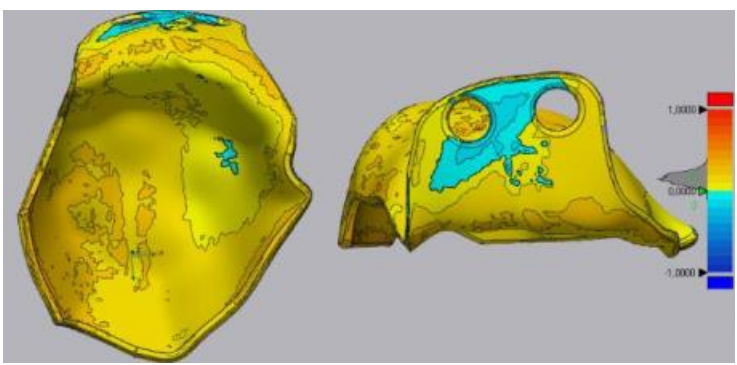

Figure 19: Results of the comparison of the scanned surface with the original CAD model

In conclusion, based on the results of the comparison, it can be seen that the calibration of the Simufact program was successful. The geometry of the preformed model approximates better to the original CAD model after being subjected to heat treatment and cut than to the model without preformation. The accuracy of the measured data always fall within the acceptable range of $\pm 0.5 \mathrm{~mm}$.

\section{Acknowledgement}

The authors are grateful that this project was carried out with the full support of the National Research, Development and Innovation Office. The title of the project was "The Design and Quality Assured Manufacturing of FreeForm Metal Prostheses by Selective Laser Melting." The identification number of the project is NVKP_16-1-20160022 .

\section{REFERENCES}

[1] Willemsen, K.; Nizak, R.; Noordmans, H. J.; Castelein, R. M.; Weinans, H.; Kruyt, M. C.: Challenges in the design and regulatory approval of 3Dprinted surgical implants: a two-case series. The Lancet Digital Health, 2019, 1(4): e163-e171; DOI: 10.1016/S2589-7500(19)30067-6

[2] Summary for the EOS M290 System Advanced User Training - Level 1 https://www.eos.info

[3] D. Magyar: Testing features of models manufactured by laser additive manufacturing, Scientific Work of Students, 2018, Faculty of Transportation Engineering and Vehicle Engineering, BME Budapest University of Technology and Economics https://tdk.bme.hu

[4] Hoang, D.; Perrault, D.; Stevanovic, M.; Ghiassi, A.: Surgical applications of three-dimensional printing: a review of the current literature \& how to get started. Ann. Transl. Med., 2016, 4(23):456 DOI: 10.21037/atm.2016.12.18 